\title{
Interprofessional collaboration in the future DMJ
}

$I^{\prime}$ $n$ addition to "What do you think?" (see preceding editorial by A. Howlett, Senior Editor-inChief), this year's DMJ team, in conjunction with the DMJ Advisory Board, has decided to promote collaboration with non-physician colleagues in patient care and research.

The central theme of this issue is medical education. Thus, we at the DMJ have decided to promote two things that we believe Dalhousie Medical School identifies as vitally important: collaboration and what we have named the "Four Humours".

Collaboration is important in medical practice as present-day physicians deliver patient care in association with large interdisciplinary care teams. These teams involve other health professionals, including physiotherapists, pharmacists, and nurses, among others. Physicians also perform research with basic and clinical researchers. Such partnership in patient care and health research allows utilization of different fields of knowledge and training.

In an attempt to mimic this real-life collaboration that occurs in medical practice and research, the DMJ has decided to invite other health professions and graduate students to submit works for publication. In this issue, the Journal is proud to publish the first interdisciplinary, award-winning paper entitled "Knit Two, Purl Two: My Therapeutic Choice" by Lindsay Cameron, a Dalhousie pharmacy student.

To further promote interdisciplinary relations, we have begun development of a section of the Journal designated "The Medicine Cabinet". The Medicine Cabinet is designed to encourage collaboration between the schools of medicine and pharmacy in the field of pharmacotherapy. Pharmacotherapy is an art that must be mastered by physicians in order to avoid medication errors that contribute to drug-related morbidity and mortality. Prescribed medications commonly lead to adverse outcomes due to drug interactions, lack of drug-therapy monitoring, drug duplication, and other preventable causes. The adverse outcomes produced by medication-related problems include prolonged hospital stay, life-threatening adverse effects, increased morbidity, and even increased mortality, especially among elderly patients taking multiple drugs. Thus, in an attempt to increase the education of our future physicians about pharmacotherapy, the DMJ has decided to provide reviews of drug therapy created by medical and pharmacy students in future issues under the headline "The Medicine Cabinet". The next issue will be the first to herald this novel collaborative section.

We at the DMJ hope that in the future we can work with other professions in the same way as we have with pharmacy in creating The Medicine Cabinet; further enhancing the knowledge of our readers through interprofessional collaboration.

This issue is also the first to feature the section entitled the "Four Humours: art, literature, history, and humour". Outstanding artistic contributions, creative writing, historical accounts, and comedic pieces relating to health, medical science, and professional practice will be published in this part of the DMJ. An amusing narrative within this issue is the Obituary of P. Latelet.

With all of the aforementioned changes evoked by the DMJ team and described by Andrew Howlett and $\mathrm{I}$, the DMJ is beginning to evolve, both in terms of structure and focus. Through published collaborative papers and interdisciplinary involvement in the "Four Humours", the future Journal should begin to reflect the interests of not only medical students and practicing physicians, but all health professional students and health professions in the Maritimes.

\section{Derek Roberts \\ Junior Editor-in-Chief, DMJ}

